\title{
OVALS IN A FINITE PROJEGTIVE PLANE
}

\author{
BENIAMINO SEGRE
}

1. Let $\mathfrak{B}$ be a finite projective plane $(8, \S 17)$, i.e. a projective space of dimension 2 over a Galois field $\gamma$. We suppose that $\gamma$ has characteristic $p \neq 2$, hence order $q=p^{h}$, where $p$ is an odd prime and $h$ is a positive integer. It is well known that every straight line and every non-singular conic of $\mathfrak{P}$ then contains $q+1$ points exactly.

Using the term oval to designate any set of $q+1$ distinct points of $\mathfrak{B}$ no three of which are collinear, we shall prove the following theorem, already surmised by Järnefelt and Kustaanheimo (3) (deemed "implausible" in Math. Rev., 14 (1953), p. 1008):

THEOREM I. If $p \neq 2$, every oval of $\mathfrak{P}$ is a conic (i.e., can be represented by an equation of the second degree).

This result fills up a gap in the finite congruence axiomatics set up by Kustaanheimo (4), and has important implications if we accept the idea, advanced by Järnefelt (2), of a possible connection between the physical world and the geometry of a finite linear space (cf. also 1, 5, 6, 7).

2. Let $\mathscr{C}$ denote any given oval of $\mathfrak{P}$, and $B$ be an arbitrary point of $\mathscr{C}$. Then $\mathscr{C}$ has a tangent at $B$, uniquely defined as the line of $\mathfrak{B}$ which contains $B$ and no other point of $\mathscr{C}$; moreover, no three tangents of $\mathscr{C}$ meet at a point (7, Theorem 3 ). We begin by proving

THEOREM II. Every inscribed triangle of $\mathscr{C}$ and its circumscribed triangle are perspective.

It is not restrictive to identify the given inscribed triangle with the triangle of reference for homogeneous coordinates $\left(x_{1}, x_{2}, x_{3}\right)$ :

$$
A_{1}:(1,0,0), \quad A_{2}:(0,1,0), \quad A_{3}:(0,0,1) ;
$$

then we may denote by

$$
a_{1}: x_{2}=k_{1} x_{3}, \quad a_{2}: x_{3}=k_{2} x_{1}, \quad a_{3}: x_{1}=k_{3} x_{2}
$$

the tangents of $\mathscr{C}$ at $A_{1}, A_{2}, A_{3}$ respectively, where $k_{1}, k_{2}, k_{3}$ are three non-zero elements of the field $\gamma$. If $B:\left(c_{1}, c_{2}, c_{3}\right)$ is any of the $q-2$ points of $\mathscr{C}$ distinct from $A_{1}, A_{2}, A_{3}$, then $c_{1} c_{2} c_{3} \neq 0$; moreover, the lines $A_{1} B, A_{2} B$, $A_{3} B$ have equations of the form

$$
x_{2}=\lambda_{1} x_{3}, \quad x_{3}=\lambda_{2} x_{1}, \quad x_{1}=\lambda_{3} x_{2},
$$

Received November 30, 1954. 
where the coefficients $\lambda_{1}, \lambda_{2}, \lambda_{3}$ are distinct from $k_{1}, k_{2}, k_{3}$ respectively, as well as from zero. Since these coefficients are given precisely by

$$
\lambda_{1}=c_{2} c_{3}^{-1}, \quad \lambda_{2}=c_{3} c_{1}^{-1}, \quad \lambda_{3}=c_{1} c_{2}^{-1},
$$

they satisfy the equation

$$
\lambda_{1} \lambda_{2} \lambda_{3}=1 \text {. }
$$

Conversely, if $\lambda_{1}$ denotes any of the $q-2$ elements of $\gamma$ distinct from zero and from $k_{1}$, the line $x_{2}=\lambda_{1} x_{3}$ meets $\mathscr{C}$ at $A_{1}$ and at a further point, $B$ say, distinct from $A_{1}, A_{2}, A_{3}$; hence the coefficients $\lambda_{2}, \lambda_{3}$ in the equations $x_{3}=\lambda_{2} x_{1}$, $x_{1}=\lambda_{3} x_{2}$ of the lines $A_{2} B, A_{3} B$ are functions of $\lambda_{1}$, connected by (1), which take once each of the non-zero values of $\gamma$ distinct from $k_{2}, k_{3}$ respectively. On multiplying the $q-2$ equations (1) thus obtained, we see that

$$
\Pi^{3}=k_{1} k_{2} k_{3} \text {, }
$$

where $I$ denotes the product of the $q-1$ non-zero elements of $\gamma$; whence

$$
k_{1} k_{2} k_{3}=-1 \text {, }
$$

as it is well known $(8, \S 59)$ that $\Pi=-1$.

From the equation (2), Theorem II follows at once. In fact the points

$$
a_{2} \cdot a_{3}:\left(k_{3}, 1, k_{2} k_{3}\right), \quad a_{3} \cdot a_{1}:\left(k_{3} k_{1}, k_{1}, 1\right), a_{1} \cdot a_{2}:\left(1, k_{1} k_{2}, k_{2}\right)
$$

are joined to $A_{1}, A_{2}, A_{3}$ respectively by the lines:

$$
x_{3}=k_{2} k_{3} x_{2}, \quad x_{1}=k_{3} k_{1} x_{3}, \quad x_{2}=k_{1} k_{2} x_{1} ;
$$

by virtue of (2), these lines concur at the point $K:\left(1, k_{1} k_{2},-k_{2}\right)$, which is therefore a centre of perspective of the triangles $A_{1} A_{2} A_{3}$ and $a_{1} a_{2} a_{3}$.

3. We can now prove Theorem I. For this purpose we use the notation of $\S 2$, assuming, as it is not restrictive, that $K$ coincides with the unit point $(1,1,1)$; this is tantamount to supposing

$$
k_{1}=k_{2}=k_{3}=-1 \text {. }
$$

If $B:\left(c_{1}, c_{2}, c_{3}\right)$ is any of the $q-2$ points of $\mathscr{C}$ distinct from $A_{1}, A_{2}, A_{3}$, we denote by

$$
b: b_{1} x_{1}+b_{2} x_{2}+b_{3} x_{3}=0
$$

the tangent of $\mathscr{C}$ at it. This line contains $B$, but none of the points $A_{1}, A_{2}, A_{3}$, $a_{2} \cdot a_{3}, a_{3} \cdot a_{1}, a_{1} \cdot a_{2}$; hence, if we put

$$
\beta_{1}=b_{1}-b_{2}-b_{3}, \quad \beta_{2}=-b_{1}+b_{2}-b_{3}, \quad \beta_{3}=-b_{1}-b_{2}+b_{3},
$$

we have

$$
b_{1} c_{1}+b_{2} c_{2}+b_{3} c_{3}=0
$$

and

$$
b_{1} b_{2} b_{3} \beta_{1} \beta_{2} \beta_{3} \neq 0
$$


By virtue of Theorem II, the triangles $B A_{2} A_{3}$ and $b a_{2} a_{3}$ are perspective; this-as is immediately seen-is expressed algebraically by the equation

$$
\left|\begin{array}{ccc}
c_{3}-c_{2} & c_{1}+c_{3} & -c_{1}-c_{2} \\
b_{1}-b_{3} & b_{2} & 0 \\
b_{1}-b_{2} & 0 & b_{3}
\end{array}\right|=0,
$$

i.e., on suppressing the non-zero factor $\beta_{1}$ :

$$
b_{2}\left(c_{1}+c_{2}\right)=b_{3}\left(c_{1}+c_{3}\right) .
$$

Likewise, the consideration of the inscribed triangles $B A_{3} A_{1}, B A_{1} A_{2}$ and their circumscribed triangles gives:

$$
b_{3}\left(c_{2}+c_{3}\right)=b_{1}\left(c_{2}+c_{1}\right), \quad b_{1}\left(c_{3}+c_{1}\right)=b_{2}\left(c_{3}+c_{2}\right) .
$$

The last three equations imply:

$$
b_{1}: b_{2}: b_{3}=\left(c_{2}+c_{3}\right):\left(c_{3}+c_{1}\right):\left(c_{1}+c_{2}\right) ;
$$

hence from (3), using also (4) and the hypothesis $p \neq 2$, we deduce the equality

$$
c_{2} c_{3}+c_{3} c_{1}+c_{1} c_{2}=0 \text {. }
$$

This equality means that each of the $q-2$ points $B$ lies on the conic

$$
x_{2} x_{3}+x_{3} x_{1}+x_{1} x_{2}=0 \text {. }
$$

Since this conic obviously contains in addition the three points $A_{1}, A_{2}, A_{3}$, and its points are precisely $q+1$ in number, thus $\mathscr{C}$ must coincide with it, which proves Theorem I.

4. We remark, in conclusion, that Theorem I does not hold on a finite plane of characteristic $p=2$, if $q>4$. For, as it is well known, the $q+1$ tangents of a non-singular conic then meet at a point; this point and $q$ of the $q+1$ points of the conic constitute an oval, which, however, is clearly not a conic.

\section{REFERENCES}

1. G. Järnefelt, A plane geometry with a finite number of elements, Verröf. Finnischen Geodatischen Inst., 36 (1949), 71-80.

2. - - Reflections on a finite approximation to Euclidean geometry, Ann. Acad. Sci. Fennicae (A, I), 96 (1951), 43 pp.

3. G. Järnefelt and P. Kustaanheimo, An observation on finite geometries, Den 11te Skandinaviske Matematikerkongress, Trondheim (1949), 166-182.

4. P. Kustaanheimo, A note on a finite approximation of the Euclidean plane geometry, Soc. Sci. Fenn. Comm. Phys. Math., 15, n. 19 (1950), 11 pp.

5. - - On the fundamental prime of a finite world, Ann. Acad. Sci. Fennicae (A, I), 129 (1952), 7 pp.

6. P. Kustaanheimo and B. Qvist, On differentiation in Galois fields, Ann. Acad. Sci. Fennicae (A, I), 137 (1952), 12 pp.

7. B. Qvist, Some remarks concerning curves of the second degree in a finite plane, Ann. Acad. Sci. Fennicae (A, I), 134 (1952), 27 pp.

8. B. Segre, Lezioni di geometria moderna, vol. 1 (Bologna, 1948).

University of Rome 